\title{
Optimal path shape for range-only underwater target localization using a Wave Glider
}

\author{
The International Journal of \\ Robotics Research \\ $1-16$ \\ (C) The Author(s) 2018 \\ Article reuse guidelines: \\ sagepub.com/journals-permissions \\ DOI: $10.1177 / 0278364918802351$ \\ journals.sagepub.com/home/ijr
}

(S)SAGE

\author{
Ivan Masmitja ${ }^{1}$, Spartacus Gomariz ${ }^{1}$, Joaquin Del-Rio ${ }^{1}$, Brian Kieft ${ }^{2}$, \\ Tom O'Reilly', Pierre-Jean Bouvet ${ }^{3}$ and Jacopo Aguzzi ${ }^{4}$
}

\begin{abstract}
Underwater localization using acoustic signals is one of the main components in a navigation system for an autonomous underwater vehicle (AUV) as a more accurate alternative to dead-reckoning techniques. Although different methods based on the idea of multiple beacons have been studied, other approaches use only one beacon, which reduces the system's costs and deployment complexity. The inverse approach for single-beacon navigation is to use this method for target localization by an underwater or surface vehicle. In this paper, a method of range-only target localization using a Wave Glider is presented, for which simulations and sea tests have been conducted to determine optimal parameters to minimize acoustic energy use and search time, and to maximize location accuracy and precision. Finally, a field mission is presented, where a Benthic Rover (an autonomous seafloor vehicle) is localized and tracked using minimal human intervention. This mission shows, as an example, the power of using autonomous vehicles in collaboration for oceanographic research.
\end{abstract}

\section{Keywords}

target localization, underwater, autonomous vehicle, acoustic, range only, single beacon, marine robotics

\section{Introduction}

Oceanographic research is an important factor to understand today's most important phenomena, such as climate change. Different technologies have been developed over recent years to study our oceans, these technologies go from space to the deepest oceans, where the focus has been centered on multi-vehicle cooperation. In this field, rangeonly and single-beacon underwater target localization using acoustic modems is a key factor.

One of the main challenges in oceanographic research lies in underwater positioning. Owing to the large attenuation of radio waves in water, it is well known that GPS signals are not suitable underwater. Therefore, different methods and architectures have been developed using acoustic signals, which have better a underwater performance, such as long baseline (LBL), ultra short baseline (USBL), and GPS intelligent buoys (GIBs). Usually, the range between two transponders is computed with knowledge of the time of flight (TOF) of a transmitted signal (and the sound speed in water), then these ranges are used to calculate the position of the sound source. Each of these systems has its own application as a function of the project's necessities and constraints. For example, the LBL system offers the best precision and accuracy, but with high deployment and maintenance costs. These costs can be somewhat reduced by GIB systems, which use surface buoys instead of sea-floor nodes. If the main goal is to reduce the set up time, the best option is a USBL system, but with less accuracy than the other methods.

On other hand, some studies have focused on singlebeacon localization methods to reduce the deployment costs (e.g. Alcocer, 2010; Olson et al., 2006; Quenzer and Morgansen, 2014; Vallicrosa et al., 2014). The main idea behind this architecture is to use an autonomous vehicle as a mobile landmark to compute the position of an

\footnotetext{
${ }^{1}$ SARTI Research Group, Electronics Department, Universitat Politecnica de Catalunya,Barcelona, Spain

${ }^{2}$ Monterey Bay Aquarium Research Institute (MBARI), California, USA

${ }^{3}$ Underwater Acoustics Lab., ISEN Brest YNCREA Ouest, France

${ }^{4}$ Marine Science Institute (ICM), Consejo superior de Investigaciones

Cientificas (CSIC), Barcelona, Spain
}

\section{Corresponding author:}

Ivan Masmitja, SARTI Research Group, Electronics Department, Universitat Politecnica de Catalunya, Rambla Exposicio 24, 08800 Vilanova i la Geltru (Barcelona), Spain.

Email: ivan.masmitja@upc.edu 
underwater target, which, while moving in the area, takes some ranges between the target and itself to triangulate the target's position. The interest in this methodology has increased over recent years, as a consequence of the necessity to reduce localization costs, and find new techniques to localize and track multiple nodes in underwater acoustic networks (UWANs), Han et al. (2013), or in fleets of autonomous underwater vehicles (AUVs), where all the nodes have their own acoustic communication modem, which can be used to determine the ranges from other nodes on the grid. For example, this methodology is used in the MORPH EC FP7 project (Kalwa et al., 2016) as explained in Furfaro and Alves (2014). The authors presented a system called distributed long baseline (DLBL), where highly synchronized modems from EvoLogics in a four-node network composed of AUVs were used.

In contrast, this technique has also been used in singlenode architectures. For example, it is used in applications such as simultaneous localization and mapping (SLAM) and aiding in AUV navigation (Bayat and Aguiar, 2013; Newman and Leonard, 2003; Tan et al., 2014), and AUV homing as well (Vaganay et al., 2000; Vallicrosa et al., 2014). Finally, single-beacon localization using autonomous vehicles as a moving landmark can also be used for target positioning and tracking in large areas without the fixed beacons' constraints. As an example, in Clark et al. (2013) a method was presented for tracking and following a tagged Leopard shark.

However, the range-only single-beacon approach has its particular challenges, such as path characterization (path shape, number of points, and maximum range) or performance evaluation (accuracy and reliability). All of these parameters must be evaluated under different circumstances and setup characteristics.

In the literature, different papers about observability (which introduces some restrictions in paths and maneuvers) can be found; for example, Hinson et al. (2013) derived that the best trajectory is to do turning motions around the beacon, and Quenzer and Morgansen (2014) used a similar approach with a surface vehicle following three AUVs. On other hand, Moreno-Salinas et al. (2016) presented a complete study to determine the optimal sensor placement for acoustic underwater target positioning with range-only measurements. Other works have focused on algorithms and their improvement under specific circumstances, such as Ramezani et al. (2013), who improved a recursive algorithm for target localization in an isogradient sound speed profile. However, all these works are mathematical developments and only show some simulations.

Fallon et al. (2010) have studied cooperative AUV navigation using surface vehicles, which use acoustic ranges as navigation aids. They studied three filtering and smoothing techniques, the extended Kalman filter (EKF), the particle filter (PF), and the nonlinear least squares (NLS), where the NLS yielded better accuracy. Experiments and field tests had been conducted in a shallow water environment. Posterior studies conducted by Webster et al. (2012) showed the performance of the centralized extended Kalman filter (CEKF) to improve the dead-reckoning navigation systems, using acoustic ranges from a surface vehicle as a navigation aid. Moreover, they showed different experiments in a deep water area. In both studies, they used the Woods Hole Oceanographic Institution (WHOI) micro-modems (Freitag et al., 2005). However, whereas their studies are extended and completed in the use of acoustic range as navigation aids, more studies are needed to characterize range-only and single-beacon target localization method, e.g. to find the best range distances or path shapes.

Finally, in other works such as those of Olson et al. (2006) and McPhail and Pebody (2009), the authors presented some field test results to localize an underwater target using range-only methods, but in their case, they do not present a general study to find the best parameters for target localization.

The work presented in this paper shows how to determine the optimal parameters of the range-only and singlebeacon target localization method. In addition, results of simulations and sea tests to demonstrate the good performance of a Wave Glider used as a single-beacon LBL system for target localization are presented. This method can be used in a wide range of applications using the long-duration, autonomous navigation, and computational characteristics of Wave Glider applications.

(i) Target localization in a benthic zone:

- instruments on seabed, which may be stationary or moving (e.g. slowly sliding down a submarine canyon, or on a Benthic Rover);

- low motion tagged benthic marine species.

(ii) Target localization in a pelagic zone:

- drifter buoys;

- AUVs;

- low motion tagged pelagic marine species.

Preliminary studies were presented in Masmitja et al. (2016a), where both simulations and field test results were shown under different circumstances such as circular radius and offsets. However, the field results in the case of different offsets did not coincide with the simulations with the same accuracy as in the radius case. In this paper, a more accurate random error model, which was described in Masmitja et al. (2016b), and a systematic error are studied to increase the simulations' accuracy. Finally, more cases such as path shape, time, and power consumption are presented to have a complete study.

This work is structured as follows. In Section 2 the optimal path shape is developed analytically. In Section 3 the range-only target localization algorithms are presented. Section 4 describes the simulations conducted to study the best parameters for underwater target localization. In Section 5 different field tests and their comparison with simulations are presented. To conclude, a real mission, where a Wave Glider was used to find a Benthic Rover, is 
explained in Section 6. Finally, the discussion and conclusions are addressed in Sections 7 and 8, respectively.

\section{Optimal path shape}

The relationship between the sensor location and the accuracy that can be achieved in measurement estimation has been widely studied, see Ucinski (2004) and the references therein. The potential areas that are faced with the sensorlocation problem include environmental monitoring, surveillance, and meteorology.

In general, the computation of the optimal sensor configuration can be carried out by examining the corresponding Cramer-Rao bound (CRB) or its Fisher information matrix (FIM) as is well known (Trees et al., 2013). In an estimation problem, where a set of noisy observations are used to estimate a certain parameter of interest, the CRB sets the lowest bound on the covariance matrix that is asymptotically achievable by any unbiased estimation algorithm.

Therefore, because the CRB is calculated from the inverse of the FIM of the likelihood function, one can use both to find the optimal sensor configuration. At this point, the determinant of the FIM is used as a performance indicator, where maximizing this quantity yields the most appropriate sensor formation geometry. For example, MorenoSalinas et al. (2016) used this method to find the optimal sensors' locations of an underwater sensor network to find a target using their ranges, and Kaune et al. (2011) derived the target's localization accuracy using time difference of arrivals (TDOAs) measurements on different sensor geometry scenarios. In this paper, similar approaches are used, where the optimal path shape can be derived taking into consideration that each sensor's position is where the Wave Glider will obtain a new range measurement from the target. This method can be called range-only and singlebeacon target localization.

Therefore, following standard procedures, the FIM corresponding to the problem of range-based target positioning can be computed from the likelihood function

$p\left(\boldsymbol{z} \mid \boldsymbol{p}_{T}\right)=\frac{1}{(2 \pi)^{m / 2}|\boldsymbol{R}|^{1 / 2}} \exp \left\{-\frac{1}{2}\left(\boldsymbol{z}-\boldsymbol{r}\left(\boldsymbol{p}_{T}\right)\right) \boldsymbol{R}^{-1}\left(\boldsymbol{z}-\boldsymbol{r}\left(\boldsymbol{p}_{T}\right)\right)\right\}$

where $m$ is the number of measurements, $\boldsymbol{p}_{T}$ is the target's position, $\boldsymbol{z}=\left[z_{1}, \ldots, z_{m}\right]^{\mathrm{T}}$ are the measured ranges, $\boldsymbol{r}\left(\boldsymbol{p}_{T}\right)$ are the true ranges between each position of the Wave Glider and target, and $\boldsymbol{R}$ are the covariance matrix. In the particular case that $\boldsymbol{R}=\sigma^{2} \boldsymbol{I}_{m}$ (where $\boldsymbol{I}_{m}$ is the identity matrix), taking the logarithm of (1), computing its derivative with respect to $\boldsymbol{p}_{T}$, and taking its expected value, the FIM can be expressed as

$$
\mathrm{FIM}=\frac{1}{\sigma^{2}} \nabla \boldsymbol{r}\left(\boldsymbol{p}_{T}\right)^{\mathrm{T}} \nabla \boldsymbol{r}\left(\boldsymbol{p}_{T}\right)
$$

For notational simplicity, and without loss of generality, hereinafter the target is considered to be placed at the origin of the inertial coordinate frame. Consequently, (2) can be rewritten as

$$
\begin{gathered}
\mathrm{FIM}=\frac{1}{\sigma^{2}} \sum_{i=1}^{m}\left[\begin{array}{ccc}
\left(\frac{\partial r_{i}\left(\boldsymbol{p}_{T}\right)}{\partial p_{T x}}\right)^{2} & \frac{\partial r_{i}\left(\boldsymbol{p}_{T}\right)}{\partial p_{T x}} \frac{\partial r_{i}\left(\boldsymbol{p}_{T}\right)}{\partial p_{T y}} & \frac{\partial r_{i}\left(\boldsymbol{p}_{T}\right)}{\partial p_{T x}} \frac{\partial r_{i}\left(\boldsymbol{p}_{T}\right)}{\partial p_{T z}} \\
\frac{\partial r_{i}\left(\boldsymbol{p}_{T}\right)}{\partial p_{T y}} \frac{\partial r_{i}\left(\boldsymbol{p}_{T}\right)}{\partial p_{T x}} & \left(\frac{\partial r_{i}\left(\boldsymbol{p}_{T}\right)}{\partial p_{T y}}\right)^{2} & \frac{\partial r_{i}\left(\boldsymbol{p}_{T}\right)}{\partial p_{T y}} \frac{\partial r_{i}\left(\boldsymbol{p}_{T}\right)}{\partial p_{T z}} \\
\frac{\partial r_{i}\left(\boldsymbol{p}_{T}\right)}{\partial p_{T z}} \frac{\partial r_{i}\left(\boldsymbol{p}_{T}\right)}{\partial p_{T x}} & \frac{\partial r_{i}\left(\boldsymbol{p}_{T}\right)}{\partial p_{T z}} \frac{\partial r_{i}\left(\boldsymbol{p}_{T}\right)}{\partial p_{T y}} & \left(\frac{\partial r_{i}\left(\boldsymbol{p}_{T}\right)}{\partial p_{T z}}\right)^{2}
\end{array}\right] \\
\mathrm{FIM}=\frac{1}{\sigma^{2}} \sum_{i=1}^{m} \frac{1}{r_{i}^{2}}\left[\begin{array}{ccc}
p_{i x}^{2} & p_{i x} p_{i y} & p_{i x} p_{i z} \\
p_{i y} p_{i x} & p_{i y}^{2} & p_{i y} p_{i z} \\
p_{i z} p_{i x} & p_{i z} p_{i y} & p_{i z}^{2}
\end{array}\right]
\end{gathered}
$$

where $\boldsymbol{p}_{i}=\left[p_{i x}, p_{i y}, p_{i z}\right]^{\mathrm{T}}$ for $i \in\{1, \ldots, m\}$ is the position of the $i$ th ranging Wave Glider's position, and $r_{i}$ the actual distance between target $\boldsymbol{p}_{T}$ and the $i$ th Wave Glider's position.

The $\log \mid$ FIM $\mid$ function is used to define the optimal FIM, which provides the maximum FIM determinant for simplicity reasons. Then, its derivatives with respect to the norms of the vectors and with respect to the angles have to be computed and set equal to zero to find its maximum and, consequently, the optimal path configuration. This process was derived in Moreno-Salinas et al. (2016), and therefore, here only the final result is presented, that is

$$
\mathrm{FIM}_{\text {opt }}=\frac{1}{\sigma^{2}}\left[\begin{array}{ccc}
\frac{m}{3} & 0 & 0 \\
0 & \frac{m}{3} & 0 \\
0 & 0 & \frac{m}{3}
\end{array}\right]
$$

Finally, the general conditions that must be satisfied by the Wave Glider path to be optimal can be derived by comparing the optimal FIM in (5) with the generic one in (4) as follows

$$
\begin{gathered}
\sum_{i=1}^{m} \frac{p_{i x}^{2}}{r_{i}^{2}}=\sum_{i=1}^{m} \frac{p_{i y}^{2}}{r_{i}^{2}}=\sum_{i=1}^{m} \frac{p_{i z}^{2}}{r_{i}^{2}}=\frac{m}{3} \\
\sum_{i=1}^{m} \frac{p_{i x} p_{i y}}{r_{i}^{2}}=\sum_{i=1}^{m} \frac{p_{i x} p_{i z}}{r_{i}^{2}}=\sum_{i=1}^{m} \frac{p_{i z} p_{i y}}{r_{i}^{2}}=0
\end{gathered}
$$

The above equations can be rewritten in terms of the angles that each range vector makes with the unit vector of the inertial reference frame as $\cos \left(\alpha_{i j}\right)=p_{i j} / r_{i}$ for $i \in\{1, \ldots, m\}$ and $j \in\{x, y, z\}$, obtaining

$$
\begin{aligned}
& \sum_{i=1}^{m} \cos ^{2}\left(\alpha_{i x}\right)=\sum_{i=1}^{m} \cos ^{2}\left(\alpha_{i y}\right)=\sum_{i=1}^{m} \cos ^{2}\left(\alpha_{i z}\right)=\frac{m}{3} \\
& \sum_{i=1}^{m} \cos \left(\alpha_{i x}\right) \cos \left(\alpha_{i y}\right)=\sum_{i=1}^{m} \cos \left(\alpha_{i x}\right) \cos \left(\alpha_{i z}\right) \\
&=\sum_{i=1}^{m} \cos \left(\alpha_{i z}\right) \cos \left(\alpha_{i y}\right)=0
\end{aligned}
$$




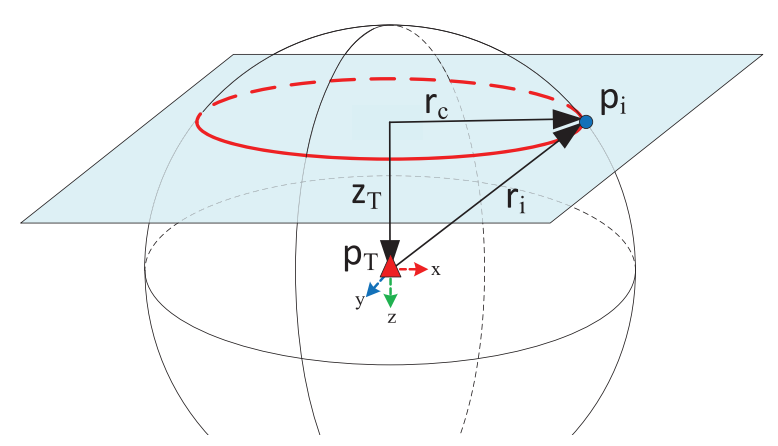

Fig. 1. Optimal geometry from the intersection between a sphere centered on the target and sea surface plane.

With this formulation, the optimal sensor configuration is described in terms of the angles between the range vectors and the inertial frame. Consequently, the ranges themselves are not an important factor in this 3D scenario, and it can be concluded that the optimal sensor configuration lies on a sphere centered on the target.

Finding a generic formulation for a 3D scenario that solves these equations to obtain the optimal geometry is not trivial, however, the scenario presented in this paper is a surface vehicle trying to localize an underwater target, which in other words means that all the sensors are placed on a plane. This situation is derived in the following subsection.

\subsection{Surface vehicle and underwater target scenario}

Considering that all the measurements are taken from a plane, which in this case is the sea surface, the optimal geometry is the intersection between a sphere centered on the target and this plane (see Figure 1). The circumference obtained (which with radius $r_{c}$ ) presents a relation between the target's depth $z_{T}$ and the ranges $r_{i}$ between the target $\boldsymbol{p}_{T}$ and the Wave Glider $\boldsymbol{p}_{i}$, which will define the optimal path that the vehicle must follow to obtain the best accuracy on the target's localization prediction problem.

Therefore, using $p_{i z}=z_{T}$, assuming that all ranges are equal, and substituting that in (6) the following relation is derived

$$
\sum_{i=1}^{m} \frac{p_{i z}^{2}}{r_{i}^{2}}=\frac{m z_{T}^{2}}{r^{2}}=\frac{m}{3} \rightarrow \frac{z_{T}}{r}=\frac{1}{\sqrt{3}}
$$

Using simply a trigonometric formulation $\left(r_{c}^{2}+z_{T}^{2}=r^{2}\right)$ the optimal path can be found, which is a circumference centered over the target with a radius equal to

$$
r_{c}=\sqrt{2} z_{T}
$$

In contrast, the difference between the optimal solution and a solution by using different values of $r_{c}$ can be derived using (10) as

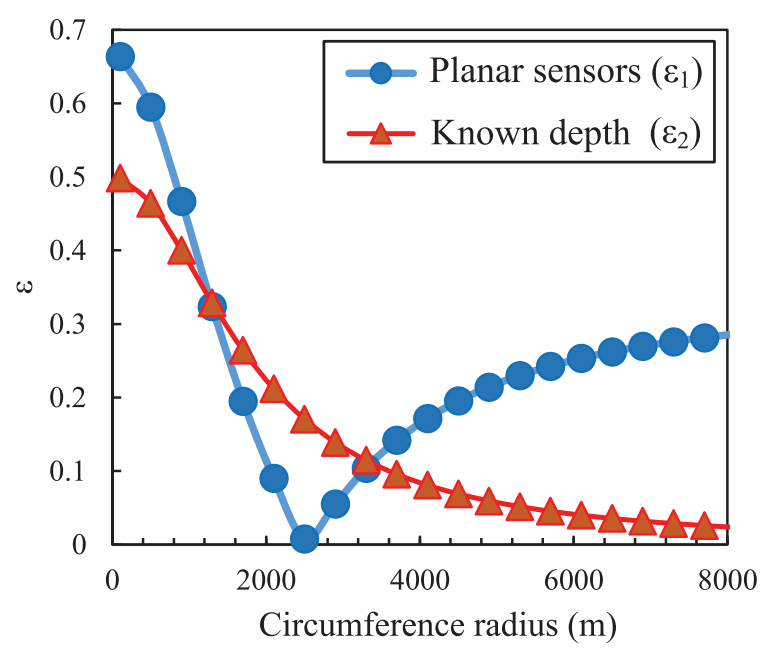

Fig. 2. Error $\epsilon$ between the optimal solution and a solution by using different values of $r_{c}$. Results obtained for both scenarios: planar sensors $\left(\epsilon_{1}\right)$ and planar sensors with a known target's depth $\left(\epsilon_{2}\right)$. These graphs should only be used as an indicator of the localization performance, they do not give the absolute accuracy reachable.

$$
\epsilon_{1}=\frac{1}{3}-\frac{z_{T}^{2}}{r_{c}^{2}+z_{T}^{2}}
$$

which can be used as an indicator of how the circumference radius affects the optimal solution, which is found when $\epsilon_{1}=0$. Figure 2 shows a specific case for a target depth equal to $1,800 \mathrm{~m}$, the optimal circumference radius is equal to $2,546 \mathrm{~m}$ can be observed.

Now, after the circumference geometry has been derived, it is necessary to find the optimal distribution of all measurements over this path. Consequently, rewriting (6) and (7) in polar coordinates, considering a unit sphere $\left(z_{T}=1 / \sqrt{3}\right.$ and $\left.r_{c}=\sqrt{2} / \sqrt{3}\right)$, and $p_{i x}=r_{c} \cos \left(\alpha_{i}\right)$, $p_{i y}=r_{c} \sin \left(\alpha_{i}\right)$ (where $\alpha_{i}$ is the projected angle of the $i$ th range vector on the $\{x, y\}$ plane), and $p_{i z}=z_{T}$, the following notation is obtained

$$
\begin{gathered}
\sum_{i=1}^{m} \cos ^{2}\left(\alpha_{i}\right)=\sum_{i=1}^{m} \sin ^{2}\left(\alpha_{i}\right)=\frac{m}{2} \\
\sum_{i=1}^{m} \cos \left(\alpha_{i}\right) \sin \left(\alpha_{i}\right)=\sum_{i=1}^{m} \cos \left(\alpha_{i}\right)=\sum_{i=1}^{m} \sin \left(\alpha_{i}\right)=0
\end{gathered}
$$

A simple and elegant solution for $\alpha_{i}$ is obtained by noticing the orthogonality relationship for sines and cosines from Fourier's analysis, which yields with the solution

$$
\alpha_{i}=\frac{2 \pi}{m} i, \quad i \in\{0, \ldots, m-1\}
$$

This means that all the measurements have to be taken uniformly distributed over the entire circumference to compute the target's most accurate position. 
Finally, it can be pinpointed that a large number of measurements $m$ yield a better estimation because $\mathrm{FIM}_{\text {opt }}=m /\left(\sigma^{6} 3^{3}\right)$ increases proportionally to $m$.

\subsection{With a known target depth}

Commonly, the target's depth can be known easily using a small and affordable sensor, which implies simple computation methods for target localization. The information of the target's depth can be sent to the Wave Glider at each range interrogation through the acoustic modems. On the other hand, if the target lies on the sea floor, the area's bathymetry can be used to compute its depth. In such situations, a 2D scenario can be derived from the $3 \mathrm{D}$ problem explained in the previous subsection knowing $z_{T}$, where instead of $r_{i}$, its projection $r_{c i}$ to the $\{x, y\}$ plane is used. Then, the FIM for the $2 \mathrm{D}$ scenario can be obtained rewriting (3) and (4) as

$$
\begin{gathered}
\mathrm{FIM}=\frac{1}{\sigma^{2}} \sum_{i=1}^{m}\left[\begin{array}{cc}
\left(\frac{\partial r_{i}\left(\boldsymbol{p}_{T}\right)}{\partial p_{T x}}\right)^{2} & \frac{\partial r_{i}\left(\boldsymbol{p}_{T}\right)}{\partial p_{T x}} \frac{\partial r_{i}\left(\boldsymbol{p}_{T}\right)}{\partial p_{T y}} \\
\frac{\partial r_{i}\left(\boldsymbol{p}_{T}\right)}{\partial p_{T y}} \frac{\partial r_{i}\left(\boldsymbol{p}_{T}\right)}{\partial p_{T x}} & \left(\frac{\partial r_{i}\left(\boldsymbol{p}_{T}\right)}{\partial p_{T y}}\right)^{2}
\end{array}\right] \\
\mathrm{FIM}=\frac{1}{\sigma^{2}} \sum_{i=1}^{m} \frac{1}{r_{c i}^{2}}\left[\begin{array}{cc}
p_{i x}^{2} & p_{i x} p_{i y} \\
p_{i y} p_{i x} & p_{i y}^{2}
\end{array}\right]
\end{gathered}
$$

where $r_{c i}^{2}=r_{i}^{2}\left(1-z_{T}^{2} / r_{i}^{2}\right)$, which yields with a FIM $_{\text {opt }}$ equal to

$$
\mathrm{FIM}_{o p t}=\frac{1}{\sigma^{2}}\left[\begin{array}{cc}
\frac{m}{2}\left(1-\frac{z_{T}^{2}}{r_{i}^{2}}\right) & 0 \\
0 & \frac{m}{2}\left(1-\frac{z_{T}^{2}}{r_{i}^{2}}\right)
\end{array}\right]
$$

In this scenario, the ratio between the slant range measurement and the target's depth plays a different role to the previous one. Here, the maximum FIM will be reached when $r_{i}^{2}$ tends to infinity; in such a case, $\mathrm{FIM}_{\text {opt }} \simeq m /\left(2 \sigma^{2}\right) \boldsymbol{I}_{2}$, which is the maximum achievable value and it is equal to the $2 \mathrm{D}$ scenario $\left(z_{T}=0\right)$. Therefore, if the target's depth is different to zero and it is known, a larger circumference radius will provide a proportionally better estimation on the target's position.

As done before, the difference between the optimal solution and a solution by using different values of the circumference's radius value can be derived using

$$
\epsilon_{2}=\frac{1}{2}-\frac{1}{2}\left(1-\frac{z_{T}^{2}}{r_{c}^{2}+z_{T}^{2}}\right)
$$

which can be used as an indicator of how the circumference's radius affects the optimal solution, which is found when $\epsilon_{2}=0$. Figure 2 shows a specific case for a target depth equal to $1,800 \mathrm{~m}$, where the optimal circumference radius tends to infinity can be observed.

Until now, all the errors that have been used were assumed constant, range independent, and with mean equal to zero, error $\sim \mathcal{N}\left(0, \sigma^{2}\right)$. Whereas this is a good approximation, which yields a tractable formulation to be studied analytically, in reality the error is more complex. Therefore, a set of different simulations with a more complex error have been carried out to study the performance, and the optimal path of range-only and single-beacon localization algorithms. However, the starting point for these simulations were the results obtained in this section.

\section{Range-only target localization algorithms}

The concept of single-beacon range-only positioning can be divided into two groups: as a navigational aid for a moving vehicle Tan et al. (2014) (group 1), or to localize a stationary or moving target Vallicrosa et al. (2014) (group 2). All these methods use a set of ranges between a target and different static nodes, known as anchor nodes or landmarks. Typically, these ranges can be obtained using TOF given the speed of sound in water. Then, the unknown underwater target position problem can be solved using trilateration, where, in general, three or more points are needed in 2D dimensions and at least four points are required in $3 \mathrm{D}$ scenarios.

In general, the navigation aid problem has received more attention in the literature (group 1) where an AUV needs to be located using a set of known transponders, as in Alcocer (2010). However, similar approaches can be used in the inverse case, where an autonomous vehicle is used to find an underwater target (group 2). The method used in this paper can be seen in Figure 3, where a range-only target localization method based on a single-beacon architecture is presented. The target's position is computed using a Wave Glider, which periodically measures the range to the underwater target, while it is moving on the surface.

Therefore, following the same notation as Alcocer (2010), the underwater target positioning vector can be defined as $\boldsymbol{p}_{T} \in \mathbb{R}^{n}$, where $n$ can be either two or three and is the space dimension of the problem. All the Wave Glider positions used in the trilateration problem can be denoted as $\boldsymbol{p}_{i} \in \mathbb{R}^{n}$ where $i \in\{1,2, \ldots, m\}$, where $m$ indicates the number of measurements carried out. Then, the ranges

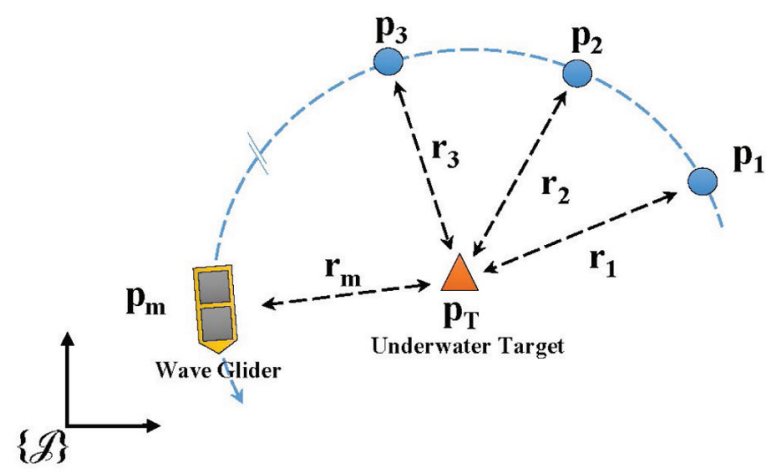

Fig. 3. Range-only single-beacon underwater target localization methodology representation, using a Wave Glider as a moving LBL. 
measured with Wave Glider between itself and the target can be expressed as

$$
\bar{r}_{i}=\left\|\boldsymbol{p}_{T}-\mathbf{p}_{i}\right\|+w_{i}, \quad i \in\{1,2, \ldots, m\}
$$

where $\left\|\boldsymbol{p}_{T}-\boldsymbol{p}_{i}\right\|=r_{i}$ is the true range, and $w_{i} \sim \mathcal{N}\left(\varepsilon, \sigma^{2}\right)$ is some non-zero mean Gaussian measurement error where $\sigma^{2}$ is the variance and $\varepsilon$ is the systematic error.

Thus, Equation (20) can be written in matrix form as $\overline{\boldsymbol{r}}=\boldsymbol{r}+\boldsymbol{w}$. In general, this non-linear, non-smooth, and overdetermined (when $m>n+1$ ) system does not have a straightforward solution. At this point, two different methodologies are used in the literature to solve the system and find the target's position through ranges (Bertsekas, 1995): linearize the function and find a closed-form least squares (LS) solution; or use an iterative minimization algorithm to minimize a cost function related to the maximum likelihood (ML) estimate.

\subsection{Closed-form LS algorithm}

As the main goal of this paper is not to compare the performance of different algorithms, a simple unconstrained least squares (ULS) algorithm is used, which was introduced in Cheung et al. (2004). However, as it will be shown, its performance is quite good.

The main idea on LS algorithms lies in a linearization of the system by using the squared range measurements to obtain a linear equation as a function of the unknown target's position $\boldsymbol{p}_{T}$ and its norm,

$$
\bar{d}=d+\xi
$$

where $\boldsymbol{d}$ is equal to the squared range $\boldsymbol{r}^{2}$, and $\boldsymbol{\xi}$ is the new measurement error as a function of $\mathbf{w}$ and $\boldsymbol{r}$. In this case, it is not obvious that $\boldsymbol{\xi} \sim \mathcal{N}\left(\varepsilon, \boldsymbol{\sigma}^{2}\right)$ as before, and the new error is not independent to the range. However, under some circumstances this assumption is possible, for example when $r_{i} \gg \sigma_{i}$, but this assumption is not true when the vehicle is close to the target. See Alcocer (2010) for more information. However, from hereafter it is assumed an error that is independent to the range and its square, which is true as the ranges used will be much larger than the error itself.

On the other hand, when all the points used to compute the underwater target position are coplanar (e.g. in the same $z$-plane), which in this case is on the sea surface, a 2D formulation can be used. The square ranges are defined by

$$
\begin{aligned}
d_{i} & =\left\|\boldsymbol{p}_{T}-\boldsymbol{p}_{i}\right\|^{2} \\
& =\left(p_{T x}-p_{i x}\right)^{2}+\left(p_{T y}-p_{i y}\right)^{2}+\left(p_{T z}-p_{i z}\right)^{2} \\
& =\left\|\boldsymbol{p}_{T}^{\prime}-\boldsymbol{p}_{i}^{\prime}\right\|^{2}+z_{T}^{2} \\
& =\boldsymbol{p}_{i}^{\prime T} \boldsymbol{p}_{i}^{\prime}-2 \boldsymbol{p}_{i}^{\prime T} \boldsymbol{p}_{T}^{\prime}+\left\|\boldsymbol{p}_{T}^{\prime}\right\|^{2}+z_{T}^{2}
\end{aligned}
$$

where $\boldsymbol{p}^{\prime}$ represents the projection of $\boldsymbol{p}$ on the $\{x, y\}$ plane, and $z_{T}$ is the target depth. This equation can be formulated in a matrix form as

$$
\boldsymbol{d}=\delta\left(\boldsymbol{P}^{T} \boldsymbol{P}^{\prime}\right)-2 P^{\prime} \boldsymbol{p}_{T}^{\prime}+\left(\left\|\boldsymbol{p}_{T}^{\prime}\right\|^{2}+z_{T}^{2}\right) 1_{m}
$$

where $\boldsymbol{P}=\left[\boldsymbol{p}_{1}, \ldots, \boldsymbol{p}_{m}\right] \in \mathbb{R}^{n x m}$, and $\delta$ is defined as the diagonal of the matrix.

The unknown scalar terms $\left\|\boldsymbol{p}_{T}^{\prime}\right\|^{2}+\delta_{z}^{2}$ are multiplying the vector of ones $1_{m}$. Therefore, this unknown term can be deleted by multiplying both sides of the equation by matrix M, which has $1_{m}$ in its null space, obtaining

$$
\boldsymbol{M d}=\boldsymbol{M} \delta\left(\boldsymbol{P}^{\prime T} \boldsymbol{P}^{\prime}\right)-2 \boldsymbol{M}{P^{\prime T}}^{\prime}=\boldsymbol{M} \boldsymbol{d}^{\prime}
$$

Consequently, the square range in two dimensions is the same as in three dimensions and the same algorithm can be used. In this situation, the depth of the target is not necessary to obtain its $(x, y)$ position. Therefore, the depth can be computed using Pythagoras' theorem. Finally, Equation (23) can be written as a linear system with form $\boldsymbol{A} \boldsymbol{\theta}=\boldsymbol{b}+\boldsymbol{\xi}$, which can be solved by minimizing as small as possible the length of the error, with solution $\boldsymbol{A}^{\mathrm{T}} \boldsymbol{A} \widehat{\boldsymbol{\theta}}=\boldsymbol{A}^{\mathrm{T}} \boldsymbol{b}$. Therefore, the target position estimation is

$$
\widehat{\boldsymbol{p}}_{T}=\boldsymbol{N}\left(\boldsymbol{A}^{\mathrm{T}} \boldsymbol{A}\right)^{-1} \boldsymbol{A}^{\mathrm{T}} \boldsymbol{b}
$$

where

$$
\begin{gathered}
\boldsymbol{N}=\left[\begin{array}{ll}
\boldsymbol{I}_{n} & 0
\end{array}\right] \\
\boldsymbol{A}=\left[\begin{array}{cc}
2 \boldsymbol{p}_{1}^{\mathrm{T}} & -1 \\
\vdots & \vdots \\
2 \boldsymbol{p}_{m}^{\mathrm{T}} & -1
\end{array}\right] \\
\boldsymbol{b}=\left[\begin{array}{c}
\left\|\boldsymbol{p}_{1}\right\|^{2}-\bar{d}_{1} \\
\vdots \\
\left\|\boldsymbol{p}_{m}\right\|^{2}-\bar{d}_{m}
\end{array}\right] \\
\boldsymbol{\theta}=\left[\begin{array}{c}
\boldsymbol{p}_{T} \\
\left\|\boldsymbol{p}_{T}\right\|^{2}
\end{array}\right]
\end{gathered}
$$

\subsection{Iterative minimization algorithm}

The main goal of this method is to use the maximum likelihood estimation (MLE), a statistical technique to compute the value that maximizes the similarity between selected values and observed data, which come with an unknown probability density function. For a normal distribution and using the log-likelihood function, which is a continuous strictly increasing function over the range of the likelihood, the log-likelihood can be written as

$$
\begin{aligned}
\log \mathcal{L}\left(\boldsymbol{p}_{T}\right) & =-\frac{m}{2} \log 2 \pi-\frac{1}{2} \log |\boldsymbol{R}|-\frac{1}{2}(\overline{\boldsymbol{r}}-\boldsymbol{r})^{\mathrm{T}} \boldsymbol{R}^{-1}(\overline{\boldsymbol{r}}-\boldsymbol{r}) \\
& =K-\frac{1}{2}(\overline{\boldsymbol{r}}-\boldsymbol{r})^{\mathrm{T}} \boldsymbol{R}^{-1}(\overline{\boldsymbol{r}}-\boldsymbol{r})
\end{aligned}
$$

where $\boldsymbol{R}$ is a diagonal matrix, the values of which are the measurement error covariance $\sigma^{2}$. Then the MLE can be found by solving the optimization problem $\widehat{\boldsymbol{\theta}}=\underset{p_{T}}{\arg \min } f\left(\boldsymbol{p}_{T}\right)$, where the cost function is 


$$
f\left(\boldsymbol{p}_{T}\right):=\frac{1}{2}(\overline{\boldsymbol{r}}-\boldsymbol{r})^{\mathrm{T}} \boldsymbol{R}^{-1}(\overline{\boldsymbol{r}}-\boldsymbol{r})
$$

In general, this cost function is non-linear because of the square root that defines the range measurements, therefore there is no closed-form solution. However, an iterative method can be used to solve this minimization problem, such as negative gradient descent or Newton's methods. Only the final formulation is presented in this paper to reduce its length, for detailed development see Alcocer (2010) and Bayat and Aguiar (2013).

To use these two iterative minimization methods the cost function gradient and its Hessian must be calculated, obtaining

$$
\nabla f\left(\boldsymbol{p}_{T}\right)=-\boldsymbol{C} \delta(\boldsymbol{r})^{-1} \boldsymbol{R}^{-1}(\overline{\boldsymbol{r}}-\boldsymbol{r})
$$

and

$$
\nabla^{2} f\left(\boldsymbol{p}_{T}\right)=-\boldsymbol{C} \delta(\boldsymbol{r})^{-2} \boldsymbol{R}^{-1} \delta(2 \boldsymbol{r}-\overline{\boldsymbol{r}}) \boldsymbol{C}^{\mathrm{T}}+\boldsymbol{\alpha}^{\mathrm{T}} \delta(\boldsymbol{r})^{-1} \mathbf{1}_{m} \boldsymbol{I}_{n}
$$

where

$$
\begin{aligned}
& \boldsymbol{R}=\left[\begin{array}{ccc}
\sigma^{2} & \cdots & 0 \\
\vdots & \ddots & \vdots \\
0 & \cdots & \sigma^{2}
\end{array}\right] \\
& \boldsymbol{\alpha}=\boldsymbol{R}^{-1}(\overline{\boldsymbol{r}}-\boldsymbol{r}) \\
& \boldsymbol{C}=\left[\begin{array}{lll}
\boldsymbol{p}_{T}-\boldsymbol{p}_{1} & \cdots & \boldsymbol{p}_{T}-\boldsymbol{p}_{m}
\end{array}\right]
\end{aligned}
$$

Using the gradient of the cost function and its Hessian, the iterative minimization algorithm can be computed by Algorithm 1.

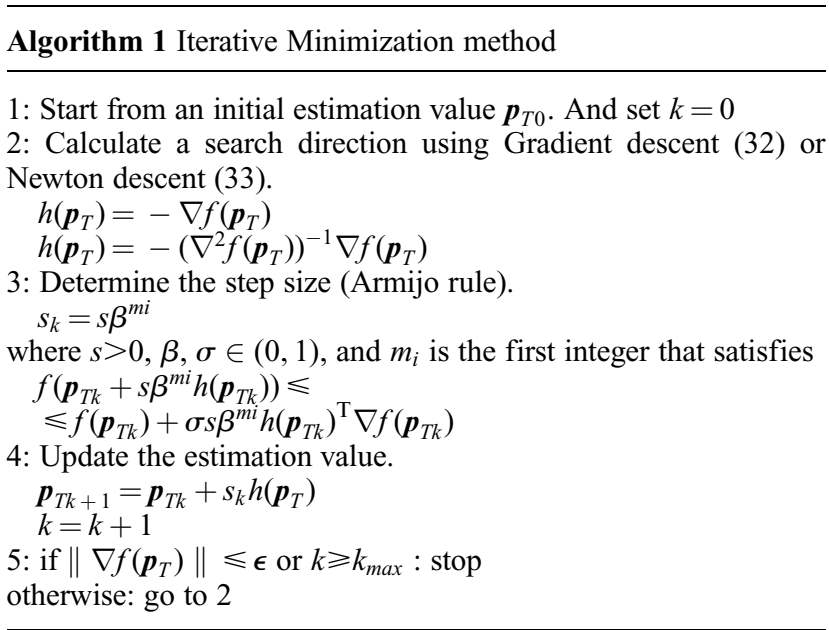

After these mathematical formulations, a set of different simulations and real tests can be conducted to characterize the performance of the system and identify the best parameters for underwater target localization using a
Wave Glider with single-range and single-beacon architecture.

\section{Simulations}

Different simulations were conducted to determine the best parameters to increase the capabilities of the acoustic positioning system. The scenario chosen is a Wave Glider on the surface conducting different paths and an underwater target at $1,800 \mathrm{~m}$ of depth to be located. Four parameters were selected: path shape, number of points needed, radius around target, and offset from target. Moreover, the performance of the derived LS and MLE algorithms was compared to the Cramer-Rao bound (CRB), which specifies the best possible performance attainable with any estimator (Rao, 2008).

The CRB theorem states that under some regular conditions of the probability density functions, the variance (37) represents the lower bound on the mean-square error of an unbiased estimator. For a scalar unbiased case, the variance of estimator $\hat{\boldsymbol{p}}_{T}$ is bounded by the Fisher information $I\left(\hat{\boldsymbol{p}}_{T}\right)$ as

$$
\operatorname{var}\left(\hat{\boldsymbol{p}}_{T}\right) \geqslant \frac{1}{I\left(\hat{\boldsymbol{p}}_{T}\right)}
$$

where the Fisher information is defined by

$$
I\left(\hat{\boldsymbol{p}}_{T}\right)=-E\left[\frac{\partial^{2} \ell\left(r ; \boldsymbol{p}_{T}\right)}{\partial \boldsymbol{p}_{T}^{2}}\right]
$$

where $\ell\left(r ; \boldsymbol{p}_{T}\right)=\log \mathcal{L}\left(\boldsymbol{p}_{T}\right)$, which can be seen in (30), and $E$ denotes the expected value. Computing the second derivative of the likelihood logarithm function and its expected value, the $\mathrm{CRB}$ obtained is

$$
\operatorname{var}\left(\hat{\boldsymbol{p}}_{T}\right) \geqslant \operatorname{tr}\left[\left(\boldsymbol{C} \delta(\boldsymbol{r})^{-1} \boldsymbol{R}^{-1} \delta(\boldsymbol{r})^{-1} \boldsymbol{C}^{\mathrm{T}}\right)^{-1}\right]
$$

which can be compared with the root mean-square error (RMSE), which represents the sample standard deviation of the differences between predicted values and observed values, using the expression RMSE $=\sqrt{\operatorname{var}\left(\hat{\boldsymbol{p}}_{T}\right)}$.

Different scenarios can be computed using (39) to observe the theoretical performance of the system (a Wave Glider as an LBL system to find an underwater target at $1,800 \mathrm{~m}$ of depth). For example, Figure 4 shows the CRB using four points as landmarks to compute the target's location. This figure shows that the best accuracy and precision are obtained when the target is located in the path's centre.

However, more scenarios have been simulated to obtain a better characterization, such as path shape, radius around the target, number of points or offset from the target. All the simulations conducted for this paper have been obtained through 1000 Monte Carlo iterations, with a normal noise probability distribution, with zero mean and variance equal to 


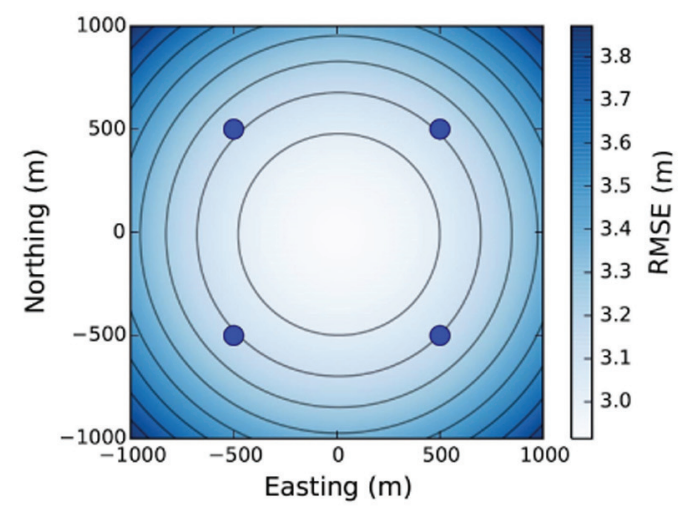

(a)

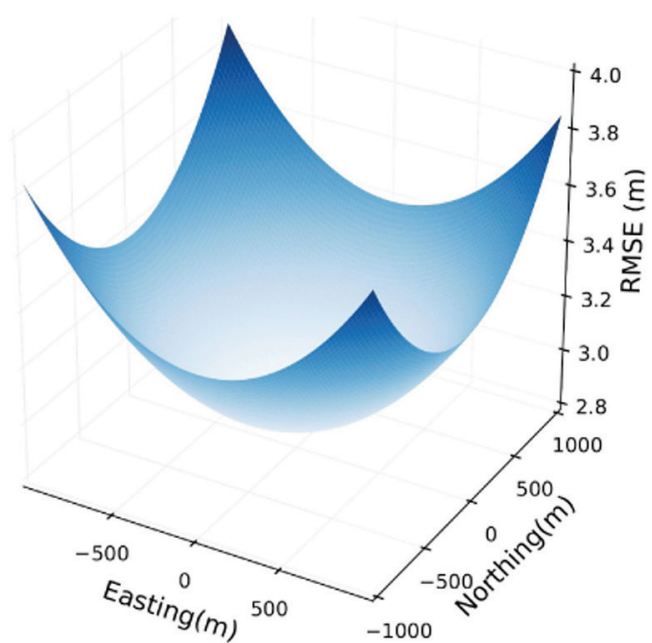

(b)

Fig. 4. CRB representation for four points as landmarks (blue dots): (a) planar representation and (b) 3D representation plots.

$$
\mathcal{U}_{c}^{2}(r)=\sum_{i=1}^{N}\left(\frac{\partial r}{\partial x_{i}}\right)^{2} \mathcal{U}^{2}\left(x_{i}\right)=\frac{1}{2} \sum_{i=1}^{N} c_{i} \mathcal{U}^{2}\left(x_{i}\right)
$$

For a better explanation of this mathematical error model and all of the parameters, see Masmitja et al. (2016b).

\subsection{Path shape}

One of the first aspects to be considered in range-only target localization is the landmark's position, or in our case the Wave Glider path shape. It is well known that the noncollinear points are mandatory, where the circular path is the optimum one as was demonstrated in the previous section. However, the best landmark positions will be determined in each case for the specific mission requirements (e.g. vehicle's use, time required, or power consumption). In this situation, path shapes other than circles could be used, where any shape can be considered as a conjunction of multiple circles and, therefore, its optimum performance is guaranteed. Figure 5 shows the RMSE evolution as a function of the path's completed ratio for four path shapes: a circle with $400 \mathrm{~m}$ of radius, and a square, a triangle, and an $\mathrm{L}$ shape with $800 \mathrm{~m}$ for each side. They all use 17 points of landmarks, which are placed on the surface of the sea, owing to the use of a Wave Glider. The dimension of these paths were chosen owing to time constraints as it is exposed in the following subsection, where they are compared with real field tests.

The RMSE for the square, the $L$, and the triangle paths is much larger than the circle path at the beginning of the path, when the path's completed ratio is less than $20 \%$. This is because all the points in these cases are still coplanar. At the end of the path the best case obtained is the square path, while the worst cases are the triangle and $L$ path. This difference is because of the ranges obtained between the Wave

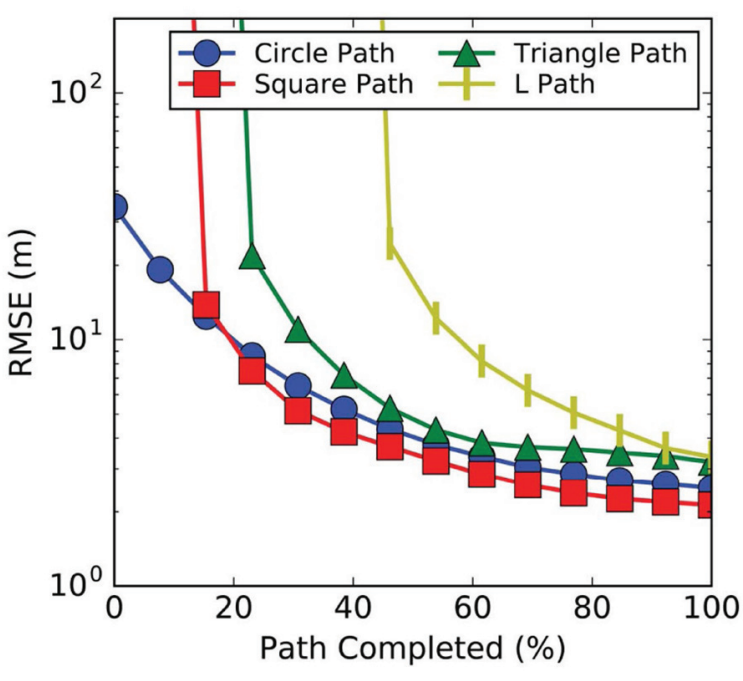

Fig. 5. RMSE evolution as a function of path completed ratio. The circle, square, triangle, and L-shape paths are represented.

Glider and the target. Longer ranges are used in the square path, and have been demonstrated in the previous sections, longer ranges cause better accuracy, where the square path can be considered as a combined set of multiple circle paths (Moreno-Salinas et al., 2016). Therefore, other aspects such as time to the path completed or power consumption should also be taken into account (for example, the Wave Glider will take more time to finish the square path than the circle path). Finally, if the path is not closed a worse performance is obtained (i.e. the square and $L$ shape), and therefore a closed path is desired, which has been demonstrated in the previous section, where all the measurements should be made equally distributed over the whole path. 


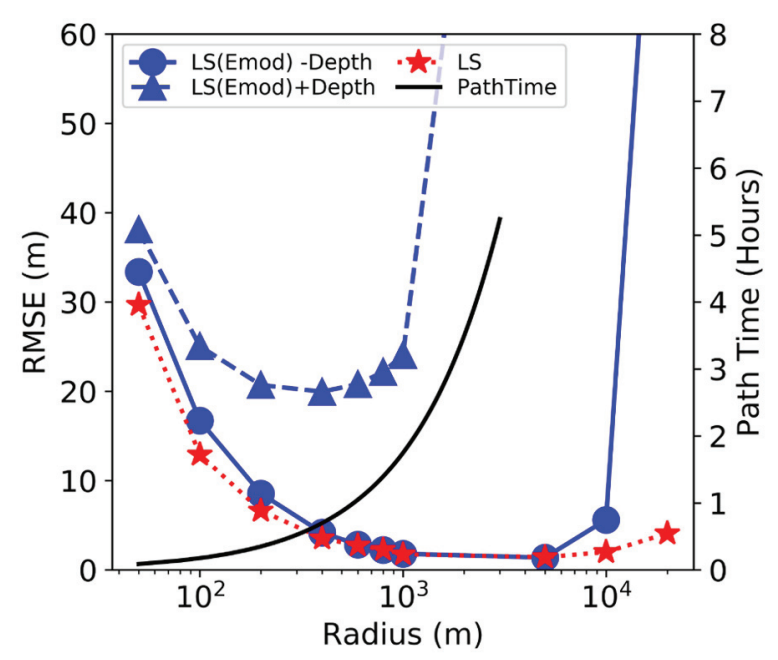

Fig. 6. The RMSE evolution, as a function of the circle radius for a target at $1,800 \mathrm{~m}$ of depth, using the LS algorithm, where $L S(E m o d)+$ Depth incorporates the range error model explained in Masmitja et al. (2016b), and shown in (40). In addition, the RMSE, when depth error is not taken into account, is plotted as $L S$ (Emod)-Depth. In both cases a $1 \%$ systematic error is added to the range measurement. These two results can be compared with a simple $\sigma=1$ error, which is used in Section 2, where the optimal radius (30) is defined. Finally, the time to complete the path is also shown (black line).

\subsection{Radius around target}

Another interesting test is to observe the behavior under different path radii centered over the target, this parameter is shown in Figure 6 where 50, 100, 200, 400, 600, 800, $1,000,5,000,10,000$, and $20,000 \mathrm{~m}$ path radii are simulated, where six points have been used as landmarks in each case. LS and ML algorithms have been compared with CRB. However, it was observed that the performance of both algorithms was very similar and very close to that of CRB. Therefore, only the LS is represented to ease understanding of the graphs. Moreover, the depth error owing to the systematic range measurement error has been treated separately (dashed line). Finally, the time necessary to finish the path has been plotted (PathTime), which can be helpful to decide the optimal circle radius.

On the other hand, a greater radius results in a lower RMSE until a specific distance where the signal-to-noise ratio (SNR) error causes an important error increase can be observed. This behavior can be derived computing the surface range $r_{s}$, which is

$$
r_{s}=\sqrt{r^{2}-\text { depth }^{2}}=\sqrt{r^{2}-(r-\alpha)^{2}}
$$

where $\alpha$ is the difference between range and depth. The error can be defined as the true value and its estimation $\varepsilon=\left(r_{s}-\bar{r}_{s}\right)$, and is

$$
\varepsilon=\sqrt{2 r \alpha-\alpha^{2}}-\sqrt{2 r(\alpha+w)-\alpha^{2}+w^{2}}
$$

where $w$ is some non-zero mean Gaussian measurement error. With (42) if $\alpha \simeq 0$ (depth and range are very similar) the error is $\varepsilon \simeq \sqrt{2 r w+w^{2}}$ and if $\alpha \simeq r$ (range is much larger than depth) the error is $\varepsilon \simeq w$ can be observed.

If the error model described in Masmitja et al. (2016b), and is shown in (40), is used (blue dotted line, LS(Emod)), the RMSE increases rapidly after a radius equal to $5,000 \mathrm{~m}$ can be observed. Therefore, the best radius will be between 1,000 and $5,000 \mathrm{~m}$. However, the time required to complete the path have to be considered, in this case the best radius can be less than 1,000 me, where the necessary time is less than 1 hour (moreover, the depth error is the most reduced).

Another method such as increasing the number of points used as landmarks can be used to reduce the RMSE instead of increasing the radius.

\subsection{Number of points}

One of the main ways to increase the precision of the system is by using more points to compute the target's position. This is the most common method to reduce the variance of any measurement with random noise, and is given by

$$
\operatorname{var}\left(z_{i}\right)=\frac{1}{n-1} \sum_{i=1}^{n}\left(z_{i}-\bar{z}\right)^{2}
$$

where $z_{i}$ are $n$ independent observations of $z$. This behavior can be seen in Figure 7, which shows the results for 4, 6, 12,24 , and 48 points. However, the optimal number of points will also be constrained by power consumption requirements, where more points will increase power consumption.

Figure 7 shows that if more points are used to compute the target's position, a better accuracy in $x$ and $y$ position is obtained, while the depth error is still equal. Therefore, the best solution is to use as many points as can be possible if the power consumption is not taken into account. This consumption can also be observed (black line) as a normalized power consumption in Figure 7, where if 50 points are used the maximum power " 1 " will be used, and otherwise, if zero points are used, the minimum power " 0 " will be used. An important difference between 20 and 50 points cannot be observed, whereas a great power consumption reduction can be obtained (note that $y$-axis is on a logarithmic scale). Therefore, around 20 points will be the desired number of landmarks to use for locating the target.

\subsection{Offset from target}

Finally, a set of simulations have been conducted to observe the RMSE with different offsets between the centre of the circular path and the underwater target, with a $400 \mathrm{~m}$ radius and 12 landmarks (Figure 8).

In this case, the introduction of a systematic error and error model $L S$ (Emod) owing to the uncertainty of water sound velocity knowledge lead to a significant difference 


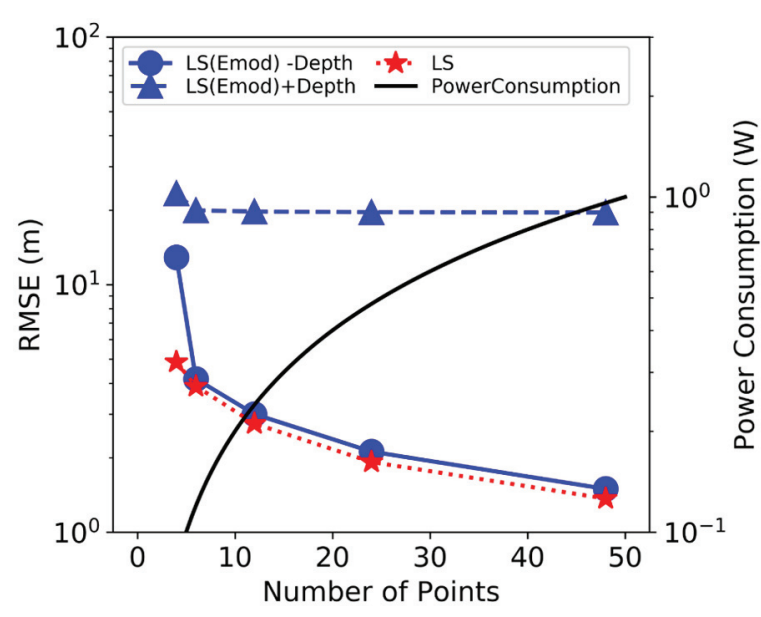

Fig. 7. RMSE evolution as a function of the number of points used to compute the target's position, for circles centered over the target (with $1,800 \mathrm{~m}$ target depth and $400 \mathrm{~m}$ of radius). The red line is the simulation result using the LS algorithm. The triangular blue dashed line is the same algorithm but with the error model $L S(E m o d)+$ Depth, and the dotted blue dashed line is the error without the depth error, LS(Emod)-Depth. In both cases, a $1 \%$ systematic error is added to each range measurement. Finally, the black line is the normalized power consumption (PowerConsumpion).

between this and the previous work shown in Masmitja et al. (2016a), where a simple random error was used (LS). Moreover, this is also different to what is derived in Section 2, and also explained in Moreno-Salinas et al. (2016), where the systematic error is not taken into account and, therefore, the error produced by circles not centered over the target is neglected. Finally, the worst effect produced by the offset is in the $x$ and $y$ RMSE can be observed in Figure 8, which in the end will rise to the same error that it is obtained with the depth measurement $(L S($ Emod $)+$ Depth $)$. Therefore, a zero offset is mandatory if a good target accuracy is desired.

\section{Real field tests}

Several sea tests have been conducted to compare and validate the algorithm's ability to locate a target, and to validate the optimal path, radius, and number of points suggested by simulations. These tests have been conducted with the Benthic Instrument Node (BIN) target placed in Monterey Bay, California, which is at $1,800 \mathrm{~m}$ of depth (in the middle of Monterey Canyon). Three groups of tests were conducted over the BIN, one to determine the best shape, another to find the best radius, and finally a third to characterize the offset effect.

\subsection{Path shape}

First, three path shapes were made with the same dimensional characteristics to observe the main differences

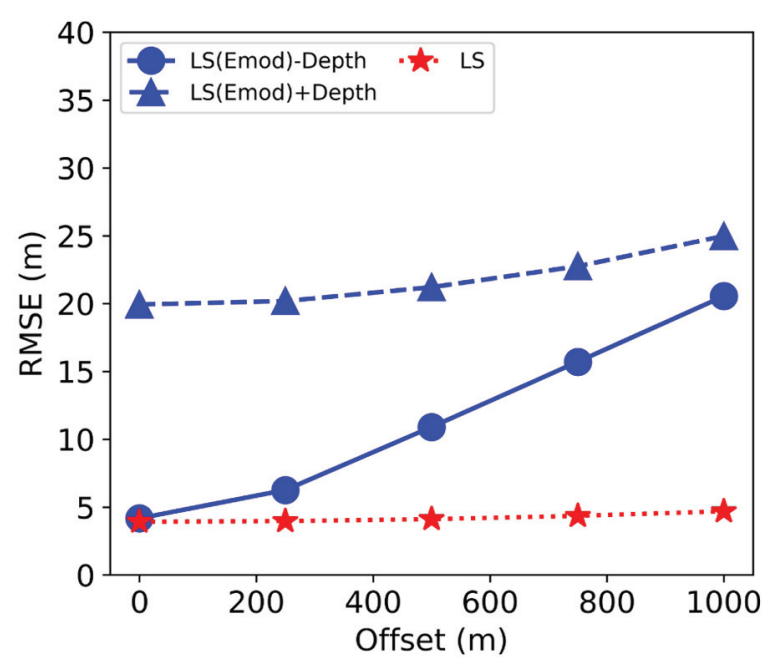

Fig. 8. RMSE evolution as a function of the offset between the circumference centre and the target, for a target at $1,800 \mathrm{~m}$ of depth. The red line is the simulation result using the LS algorithm, the triangular blue dashed line is the same algorithm but with the error model plus a systematic error of $1 \%$ $L S($ Emod $)+$ Depth, and the dotted blue dashed line is without the depth error, $L S$ (Emod)-Depth.

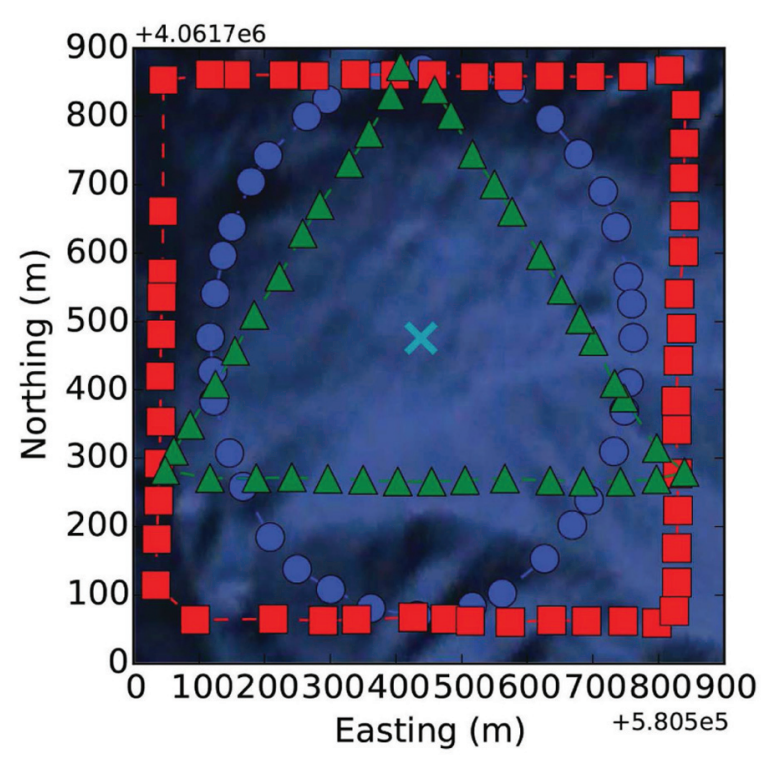

Fig. 9. Wave Glider trajectories performed over the BIN target (X) with three different paths, a square, a triangle, and a circle.

among them. These paths were a circle with $400 \mathrm{~m}$ radius, and a square and triangle with $800 \mathrm{~m}$ sides. These values have been chosen to be able to compare them with simulations. Figure 9 shows the paths obtained and Table 1 lists the main values: the target's position computed using the LS algorithm (easting, northing, and depth), the error versus the target's true position, the number of points used $\left(N_{p}\right)$, and the total of time to complete the path. The target's true position was obtained using the average value of three paths shapes with a total of 154 ranges. 
Table 1. Main results for field test 1.

\begin{tabular}{|c|c|c|c|c|c|c|c|c|}
\hline Path & Easting & Error $^{\mathrm{a}}$ & Northing & Error $^{\mathrm{a}}$ & Depth & Error $^{\mathrm{a}}$ & $N_{p}$ & Time \\
\hline Circle & $580,937.0$ & 0.9 & $4,062,175.6$ & -3.8 & $1,858.7$ & -5.1 & 36 & $56^{\prime}$ \\
\hline Square & $580,937.5$ & 1.4 & $4,062,177.4$ & -2.0 & $1,861.9$ & -1.9 & 51 & $1 \mathrm{~h} 50$ \\
\hline Triangle & $580,937.9$ & 1.8 & $4,062,176.0$ & -3.4 & $1,858.5$ & -5.3 & 39 & $55^{\prime}$ \\
\hline
\end{tabular}

${ }^{a}$ Error from target's true position was obtained using the average value of three path shapes with a total of 154 ranges. Values in meters.

Table 2. Main results for field test 2.

\begin{tabular}{rlrlrrrrr}
\hline Path & Easting & Error $^{\mathrm{a}}$ & Northing & Error $^{\mathrm{a}}$ & Depth & Error $^{\mathrm{a}}$ & $N_{p}$ & Time \\
\hline r100 & $580,922.1$ & 15.1 & $4,062,178.8$ & -1.2 & $1,860.7$ & 0.7 & 11 \\
r400 & $580,937.0$ & 0.2 & $4,062,175.6$ & 2.0 & $1,858.7$ & 2.7 & 36 & 13 \\
r800 & $580,936.1$ & 1.1 & $4,062,179.4$ & -1.8 & $1,863.8$ & -2.4 & 64 & $1 \mathrm{~h} \mathrm{57}$, \\
\hline
\end{tabular}

${ }^{a}$ Error from target's true position was obtained using the average value of the three paths' shapes from test 1 with a total of 154 ranges. Values in meters.

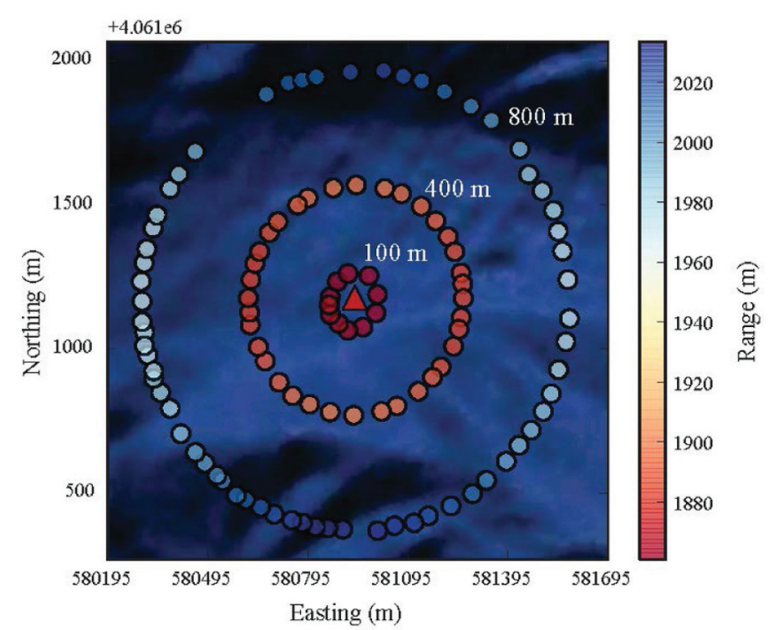

Fig. 10. Wave Glider trajectories conducted over BIN target with three different radius, which were 100,400 , and $800 \mathrm{~m}$. The ranges are also plotted using the color bar legend on the right.

In Table 1 the main results obtained during the three different path shape tests can be observed. The target localization RMSE (for $x$ and $y$ positions) obtained during these paths was approximately the same, $3.5 \mathrm{~m}$. However, a better accuracy for the square path was measured, owing to two reasons: first, the square path uses longer ranges, and this causes a better accuracy (Section 2) as it has been pinpointed in Section 4.1, where the square path can be considered as a combined set of multiple circle paths without loss of generality (Moreno-Salinas et al., 2016); second, the square path used in this field test had more points to compute the target's localization $\left(N_{p}=51\right)$, and increasing the number of points yields into the reduction of the variance of a measured value as it is highlighted in Section 4.3. Nevertheless, the square shape's time required to complete the path was around $1 \mathrm{~h}$ more than the time required for the triangle and circle paths, this reduced the viability of using this path for scenarios where speed is an important factor, for example where multiple scientific tests have to be carried out or the weather prediction is not very good. Similar results were obtained with the simulations, therefore the circle is one of the best paths among these path shapes can be concluded, owing to its speed and accuracy.

\subsection{Radius around target}

Figure 10 shows the path shapes of the second group of tests, which consist of three circles of $100 \mathrm{~m}, 400 \mathrm{~m}$ and $800 \mathrm{~m}$ of radius, all of them centered over the BIN, where the main results are shown in Table 2. Note that an $800 \mathrm{~m}$ of maximum radius was selected during the field test to reduce the time consumption, which does not compromise the accuracy, as explained in section 4.

Moreover, the ranges obtained during the field tests can be observed in Figure 10, which were around 2,020, 1,920, and $1,880 \mathrm{~m}$ for path circles with a radius equal to 800 , 400 , and $100 \mathrm{~m}$, consecutively.

To compare field test results with the simulation results, the target's position using only six equidistant points among all the ranges obtained in the field test during one circle path was computed, which allows us to choose different groups of six points and take the average value of the RMSE (represented by red circles and denoted as Real Data in Figure 11). In addition, the power trend line (red dashed line) is computed and represented to obtain a better representation of performance. The target position was computed using the LS algorithm in both simulations and field test. The LS algorithm is accurate enough compared with MLE as is explained in Section 4. Moreover, the error bars are plotted to show the standard deviation of uncertainty and the mean point $L S($ Emod), during a 1,000 run 
Table 3. Main results for field test 3 .

\begin{tabular}{|c|c|c|c|c|c|c|c|c|}
\hline Path & Easting & Error $^{\mathrm{a}}$ & Northing & Error $^{\mathrm{a}}$ & Depth & Error $^{\mathrm{a}}$ & $N_{p}$ & Time \\
\hline d0 & $580,937.0$ & 0.2 & $4,062,175.6$ & 2.0 & $1,858.7$ & 2.7 & 36 & $56^{\prime}$ \\
\hline d500 & $580,946.3$ & -9.1 & $4,062,187.1$ & -9.5 & $1,860.3$ & 1.1 & 34 & $59^{\prime}$ \\
\hline d1000 & $580,956.8$ & -19.6 & $4,062,190.2$ & -12.6 & $1,856.3$ & 5.1 & 43 & $1 \mathrm{~h} \mathrm{4}{ }^{\prime}$ \\
\hline
\end{tabular}

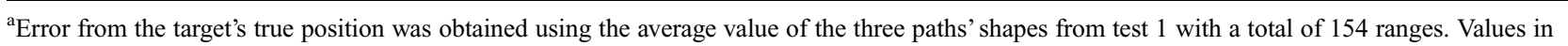
meters.

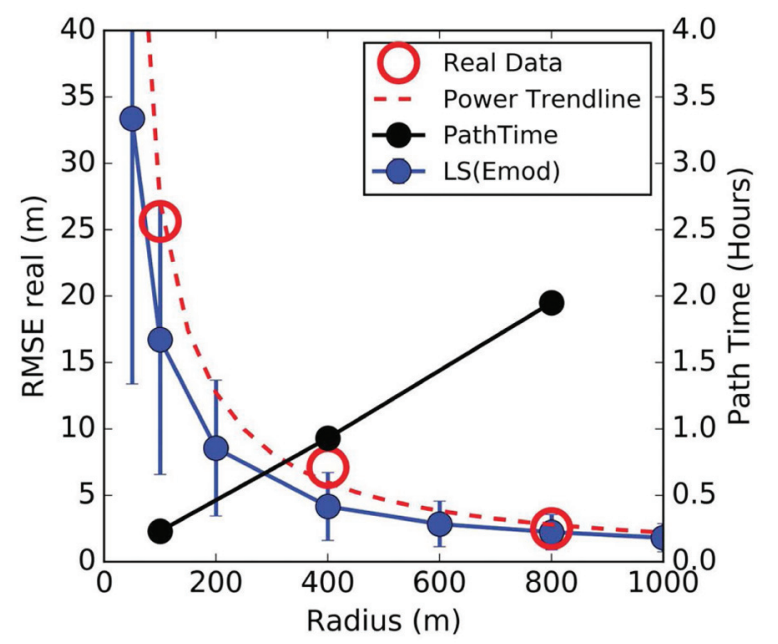

Fig. 11. Comparison of the behavior between simulation (with error model $L S(E m o d)$ ) and real data results for different radii of circle paths centered over the BIN target. Using six equidistant points to compute the target's localization and the LS algorithm. The dashed line is the power trend line computed using real data (red circles). Moreover, the time which was required to complete the path is also represented (black line).

times simulation using the error model described in Masmitja et al. (2016b).

In this graph the real data behavior is similar to the results obtained with simulations can be observed. With a radius equal or greater than $400 \mathrm{~m}$ a good performance was obtained, with a RMSE lower than $10 \mathrm{~m}$. A RMSE lower than $5 \mathrm{~m}$ can be obtained for a radius greater than $800 \mathrm{~m}$. However, the time required (PathTime) by the Wave Glider to complete the path has to be taken into account, which introduces an important limitation. For example, the Wave Glider needs almost $2 \mathrm{~h}$ to complete a circle of $800 \mathrm{~m}$ radius, while this time is reduced to $1 \mathrm{~h}$ for a radius equal to $400 \mathrm{~m}$.

\subsection{Offset from target}

Finally, a third field test was conducted over the BIN target to observe the offset's influence in the accuracy. For this purpose three paths were conducted, with distances of 0 , 500 , and 1,000 $\mathrm{m}$ between the circumference center and the target, and with a radius of $400 \mathrm{~m}$. Figure 12 shows the path shapes of this third test, where the ranges obtained are

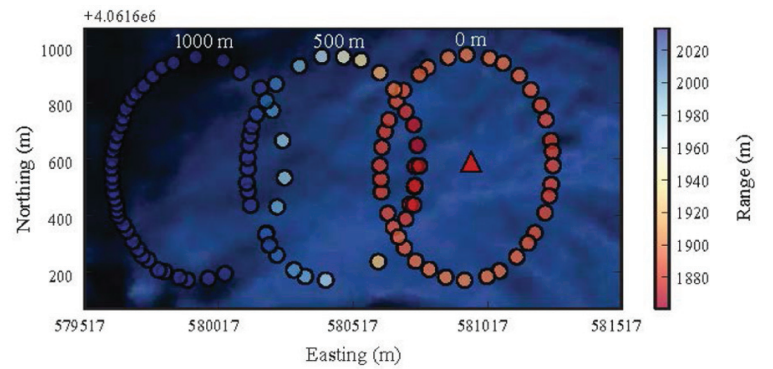

Fig. 12. Wave Glider trajectories performed over BIN target with three different offsets between target and circle centre, which were 0,500 , and $1,000 \mathrm{~m}$.

also represented using the color bar on the right. Ranges between 2,030 and $1870 \mathrm{~m}$ were obtained. Moreover, the main results are shown in Table 3.

The RMSE using different groups of six equidistant ranges to compare the field results with simulations were computed, as has done in the previous subsection. These results can be observed in Figure 13, where the similarity between both the behavior and the RMSE in real tests and simulations can be observed. Therefore, a better mathematical model than in our previous work (Masmitja et al., 2016a) has been obtained, which consisted in taking into account the systematic error and a better random error model (40).

\section{Benthic Rover mission}

Finally, a mission performed to find a Benthic Rover (McGill et al., 2007), and to know its trajectory is explained in this section. This is a final demonstration to show some of the uses of a Wave Glider as a moving LBL system. The Benthic Rover is a mobile physiology laboratory designed by Dr Ken Smith at MBARI, which slowly crawls along the seafloor. The Rover and its deployment localization can be observed in Figure 14, which also shows Monterey Bay and the MBARI laboratories' localization.

The main goal of this mission was to observe whether the moving benthic instrument was working correctly. The Rover was moving forward very slowly on the seafloor following a straight line. The initial parameters were set to a velocity equal to $5 \mathrm{~m} /$ day, following a line of $45^{\circ}$ in inclination with respect to the magnetic north. As a result, its 


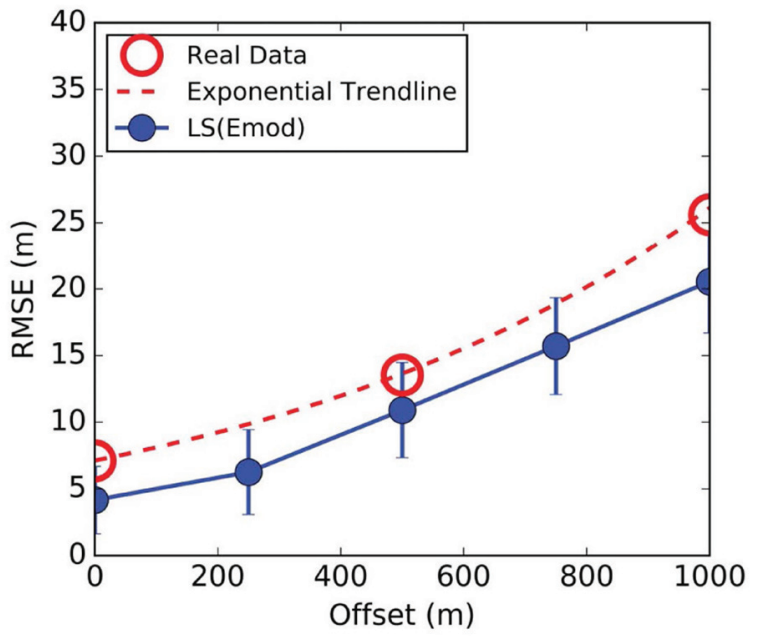

Fig. 13. Behavior comparison between simulation (with error model $L S(E m o d)$ ) and real data results for different offsets of circle paths over the BIN target. Using six equidistant points to compute the target's localization. The dashed line is the exponential trend line computed using real data.

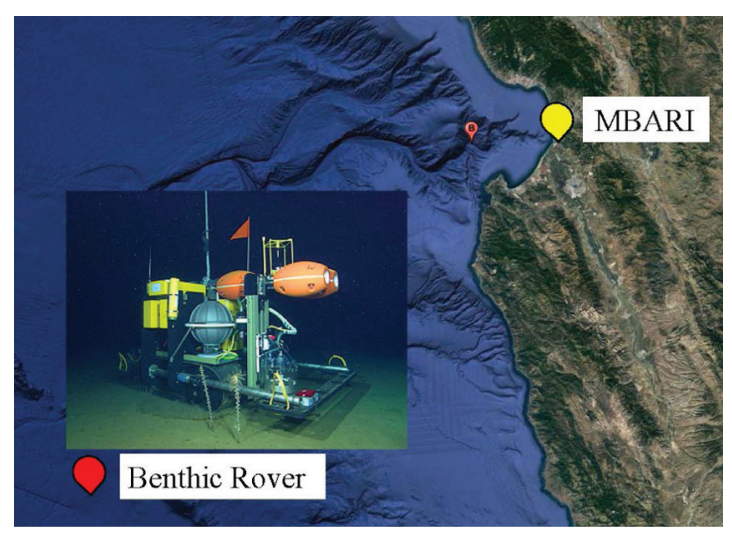

Fig. 14. Initial Benthic Rover deployment at "Station $M$ " in the North Eastern Pacific Ocean, at $34^{\circ} 50^{\prime} \mathrm{N}$ and $123^{\circ} 00^{\prime} \mathrm{W}$, a region with $4,000 \mathrm{~m}$ of depth, situated at $220 \mathrm{~km}$ west of central California coast. In addition, the MBARI localization is represented at the center of Monterey Bay.

position's estimation could be computed. Therefore, the Rover's position estimated using its initial parameters and the position founded using the Wave Glider could be compared, and used to observe whether the trajectory followed by the Rover was the programmed one.

To accomplish this objective, an initial position and two localization missions were used (as shown in Figure 15).

(a) Initial position: The Benthic Rover was deployed at geographic coordinates $35^{\circ} 7^{\prime} 59.988^{\prime \prime} \mathrm{N}$ and $123^{\circ} \mathrm{W}$, on 11 August 2015.

(b) Test 1: First localization mission conducted on 14 April 2016. In this case the Rover was localized at

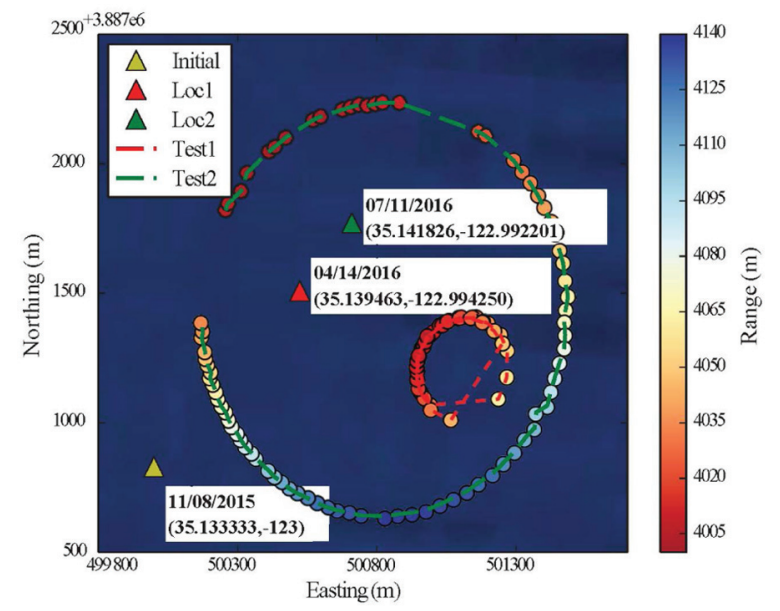

Fig. 15. The Benthic Rover's deployment position (yellow triangle), and the two missions conducted to find it (red and green triangles and circles).

$35^{\circ} 8^{\prime} 22.0668^{\prime \prime} \mathrm{N}$ and $122^{\circ} 59^{\prime} 39.3^{\prime \prime} \mathrm{W}$, which means that it had traveled $858 \mathrm{~m}$ in 158 days, with an angle of $52^{\circ}$.

(c) Test 2: Finally, a last mission conducted on 11 July 2016 , localized the Rover at $35^{\circ} 8^{\prime} 30.5736^{\prime \prime} \mathrm{N}$ and $122^{\circ} 59^{\prime} 31.9236^{\prime \prime} \mathrm{W}$. In this case, it had traveled $322 \mathrm{~m}$ in 88 days, with an angle of $55^{\circ}$, from the last known point.

Therefore, the Benthic Rover traveled $1,180 \mathrm{~m}$ in total for 246 days. This indicates a velocity of $4.8 \mathrm{~m} /$ day, which is highly close to the programmed one, obtaining an error of $40 \mathrm{~m}$ between the final estimated position and the position obtained using the Wave Glider. On the other hand, the inclination followed by the Rover was around $53.5^{\circ}$ in respect to the geodetic north. If the magnetic declination is taken into consideration, which was $13.15^{\circ}$ east in this area, the trajectory of the rover was $40.35^{\circ}$ with respect to the magnetic north, which yields an error of less than $5^{\circ}$ compared with the programmed one.

The missions performed to find and track the Benthic Rover, using a Wave Glider, shows an example of collaboration between two autonomous vehicles, with minimal human intervention. Moreover, using range-only and single-beacon methods for target localization, we are not limited to work in a specific area (as in traditional LBL systems), and we do not need to introduce more instruments (like a USBL), instead of that, standard acoustic modems can be used, which are also used to communicate and download information from underwater instruments. For these reasons, this method is interesting in terms of cost, flexibility, and consumption.

Finally, the reasons to choose the paths selected to perform this mission were twofold, the time required to complete the path and the desirable accuracy. The first test was carried out using a $200 \mathrm{~m}$ radius circle. In this case, a first 
inaccurate estimation of the Rover's position was required. Moreover, owing to other tests that had to be carried out, the time constraint was a key factor. Then, a more accurate localization was desirable during the second test and, therefore, more time was designated for the localization mission. In this case, an $800 \mathrm{~m}$ radii circle was used, which is one of the best radius in terms of accuracy and time consumption, as can be observed in the previous study explained above.

\section{Discussion}

The aim of this paper was to study and develop new procedures for underwater target localization using a Wave Glider (an AUV), which could be used as a platform in support of applications in marine, geoscientific, ecology, and archaeology, which have been increasingly used over the past 30 years (Williams et al., 2016). Here, a complete study about the best practices for underwater target localization using range-only techniques has been carried out, which includes different areas such as analytical studies, simulations, and field tests. At the same time, a real mission to find an underwater rover has been presented, where the successful collaboration between both autonomous vehicles was shown. From a methodology point of view, this work advanced the understanding of accuracy that can be achievable by using both range-only and single-beacon localization methods and an autonomous vehicle, which has been demonstrated not only numerically, but also in real tests. In this context, those advancements would contribute to expanding the use of surface vehicles, and in concrete Wave Gliders, as multipurpose platforms, which have been used widely around the world (Manley et al., 2017).

Most of the works about optimal sensor placement for underwater target localization are centered on analytical studies (Kaune et al., 2011; Moreno-Salinas et al., 2016). Whereas this is an important area of study, real tests have a great impact on the final users, which demonstrates not only in simulations but also in real missions the operability of this kind of systems. As far as the authors know, such complete study, where both theoretical and practical work is addressed, has not been conducted previously.

The initial point of this paper is the work performed by Moreno-Salinas et al. (2016), which studied the optimal sensor placement for target localization. However, whereas they work with multiple sensors, the work presented here is focused on a single sensor (which is the Wave Glider), therefore a different point of view is used. Moreover, owing to the mission's limitations, such as time and power consumptions, new different limitations have been studied. One has to take into consideration such limits before planning each missions, these are a key factor, which are really important for vehicle operators. As shown, finally a relationship between accuracy and time/power consumptions is obtained, and the mission planner must deal with that.

As a summary, the following indications should be, in general, followed before planning a mission to find the optimal path. (a) The optimal path is a circle centered over the target's position.

(b) The optimal circle's radius is:

- $r_{c}=\sqrt{2} z_{T}$ if the target's depth is unknown; or

- as large as possible if the target's depth is known.

(c) The optimal measurements distribution is equally distributed over the circle's path.

The optimal number of measurements is as large as possible.

However, as demonstrated, in some scenarios it is not possible to use these indications (e.g. when the time to complete the mission is not long enough) and, therefore, a smaller radius has to be used. Nevertheless, in the field test (for a target depth equal to $1,800 \mathrm{~m}$ ) a RMSE of less than $5 \mathrm{~m}$ had been obtained using a radius of $800 \mathrm{~m}$ instead of $1,800 \sqrt{2} \mathrm{~m}$, which is in general good enough for many missions.

In contrast, a Gaussian noise with zero mean and variance equal to $\sigma$ as range error has been used during the analytical derivation of the optimal path's shape. It was assumed that this error was range independent and equal for all range values. This procedure enables the analytical interpretation of the mathematical equations. However, the variance of the range error can be much more complex, which is determined by different parameters such as SNR, transmission frequency, weather conditions, and sea state. All these factors were discussed in Masmitja et al. (2016b). Moreover, the range error suffers from a systematic error, which is due to underwater sound speed uncertainty, which is usually difficult to measure qualitatively in situ. As a consequence, this error introduces a constant error in the range measured. This is also dependent to the range. Consequently, in the simulations that have been conducted, the range error introduced in Masmitja et al. (2016b) plus a $1 \%$ systematic error have been used to increase the similarity between simulations and the real world. It has been observed that to reduce the range error consequences, a path centered over the target is desired. However, while the error in $x$ and $y$ can be solved easily using this recommendation, with the depth error one has to be more careful. The common way to solve the depth error is by using a depth sensor, because it is easy to find a small and cheaper sensor on the market with good performance. Moreover, other methodologies can be used such as pre-calibration or path techniques to find the exact underwater speed sound or depth position (McPhail and Pebody, 2009).

Finally, the similarity among the performance of the analytical methodology used, the simulations using LS and MLE, and the field tests can be highlighted. For example, if Figures 2 and 6 are compared, in both cases a minimum error is obtained at a similar radius, which is when the $\epsilon_{1}$ and $L S$ graphics are minimum. However, if the error model plus a systematic error is used, the minimum error that is achievable is obtained much earlier, LS(Emod) + Depth . This performance is also observed in the field tests, see 
Figure 11. Similar situations can be derived in the other cases under study, such as path shape and target offset.

To conclude, the main benefit of the simulations with respect to the analytical studies is that they can give the final users the expected RMSE, instead of a simple indication of their performance. Therefore, the simulations can be used to find the accuracy that can be achievable under different conditions, such as the path shape, but also the range error estimated.

\section{Conclusions}

This work extends the study conducted in Masmitja et al. (2016a) and shows the Wave Glider's performance as a moving LBL with simulations and real sea tests. Mathematical algorithms and performance have been compared with sea tests, showing a good similarity, which corroborates the simulations conducted in this paper.

Two different algorithms have been implemented, the LS and the MLE, which have been compared through 1,000 Monte Carlo iteration simulations. The scenario implemented was a static target at 1,800 $\mathrm{m}$ depth. In this case, both algorithms show a similar performance, which is close to the CRB.

Moreover, three types of field tests have been conducted to observe the system's performance under different conditions: the path shape, the path radius, and the offset from the target. For each test three different paths have been conducted, which result in nine Wave Glider missions, more than 300 ranges, and around 10 hours of tests.

With this study the best path and its characteristics can be determined, such as the number of points, the radius, or offset, to obtain the desired target localization performance, which are a minimum number of points equal to 12 , a radius between 400 and $800 \mathrm{~m}$, and an offset as low as possible. With these parameters a RMSE less than $4 \mathrm{~m}$ can be obtained, while maintaining both low time and power consumption requirements.

Finally, it can be concluded that the Wave Glider can be used as a moving LBL to find underwater targets with a good accuracy, as demonstrated in the experimental tests and the Benthic Rover mission explained in this paper. This system has been mathematically modeled and tested under real conditions, obtaining a good performance. Therefore, this will be a new powerful tool among MBARI's equipment for future missions.

\section{Acknowledgments}

We gratefully acknowledge the support of MBARI and the David and Lucile Packard foundation. This work has been lead and carried out by members of the Tecnoterra associated unit of the Scientific Research Council through the Universitat Politécnica de Catalunya, the Jaume Almera Earth Sciences Institute and the Marine Science Institute.

\section{Funding}

This work was partially supported by the project JERICO-NEXT from the EC-H2020 (grant agreement number 654410; EvoLUL TNA sub-project). We are also grateful for the financial support from the Spanish Ministerio de Economa y Competitividad (contract TEC2017-87861-R project RESBIO and CGL2013-42557-R project INTMARSIS). The main author of this work has a scholarship (FPI-UPC) from UPC for his PhD research (agreement number 175/2015).

\section{References}

Alcocer A (2010) Positioning and Navigation Systems for Robotic Underwater Vehicles. PhD Thesis, Instituto Superior Tecnico, Universidade Tecnica de Lisboa.

Bayat M and Aguiar AP (2013) AUV range-only localization and mapping: Observer design and experimental results. In: 2013 European Control Conference (ECC), pp. 4394-4399.

Bertsekas D (1995) Nonlinear Programming. 2nd ed. Belmont, MA: Athena Scientific.

Cheung KW, So HC, Ma WK and Chan YT (2004) Least squares algorithms for time-of-arrival-based mobile location. IEEE Transactions on Signal Processing 52(4): 1121-1130.

Clark CM, Forney C, Manii E, et al. (2013) Tracking and following a tagged leopard shark with an autonomous underwater vehicle. Journal of Field Robotics 30(3): 309-322.

Fallon MF, Papadopoulos G, Leonard JJ and Patrikalakis NM (2010) Cooperative auv navigation using a single maneuvering surface craft. The International Journal of Robotics Research 29(12): 1461-1474.

Freitag L, Grund M, Singh S, Partan J, Koski P and Ball K (2005) The WHOI micro-modem: An acoustic communications and navigation system for multiple platforms. In: Proceedings of OCEANS 2005 MTS/IEEE, Vol. 2, pp. 1086-1092.

Furfaro TC and Alves J (2014) An application of distributed long baseline - node ranging in an underwater network. In: 2014 Underwater Communications and Networking (UComms), pp. $1-5$.

Han G, Xu H, Duong TQ, Jiang J and Hara T (2013) Localization algorithms of wireless sensor networks: A survey. Telecommunication Systems 52(4): 2419-2436.

Hinson BT, Binder MK and Morgansen KA (2013) Path planning to optimize observability in a planar uniform flow field. In: 2013 American Control Conference, pp. 1392-1399.

Kalwa J, Tietjen D, Carreiro-Silva M, et al. (2016) The European Project MORPH: Distributed UUV systems for multimodal, 3D underwater surveys. Marine Technology Society Journal 50(4): $26-41$.

Kaune R, Hörst J and Koch W (2011) Accuracy analysis for TDOA localization in sensor networks. In: 14th International Conference on Information Fusion, pp. 1-8.

Manley JE, Carlon R and Hine G (2017) Ten years of wave glider operations: A persistent effort. In: OCEANS 2017, Anchorage, AK, pp. 1-5.

Masmitja I, Gomariz S, Rio JD, Kieft B and O'Reilly T (2016a) Range-only underwater target localization: Path characterization. In: OCEANS 2016 MTS/IEEE, Monterey, CA, pp. 1-7.

Masmitja I, Pallares O, Gomariz S, Rio JD, Kieft B and O'Reilly $\mathrm{T}$ (2016b) Range-only underwater target localization: Error characterization. In: 21st IMEKO TC4 International Symposium, Budapest, pp. 267-271. 
McGill PR, Sherman AD, Hobson BW, Henthorn RG, Chase AC and Smith KL (2007) Initial deployments of the rover, an autonomous bottom-transecting instrument platform for longterm measurements in deep benthic environments. In: OCEANS 2007, pp. 1-7.

McPhail SD and Pebody M (2009) Range-only positioning of a deep-diving autonomous underwater vehicle from a surface ship. IEEE Journal of Oceanic Engineering 34(4): 669-677.

Moreno-Salinas D, Pascoal A and Aranda J (2016) Optimal sensor placement for acoustic underwater target positioning with range-only measurements. IEEE Journal of Oceanic Engineering 41(3): 620-643.

Newman P and Leonard JJ (2003) Pure range-only sub-sea SLAM. In: ICRA, vol. 2, pp. 1921-1926.

Olson E, Leonard JJ and Teller S (2006) Robust range-only beacon localization. IEEE Journal of Oceanic Engineering 31(4): 949-958.

Quenzer JD and Morgansen KA (2014) Observability based control in range-only underwater vehicle localization. In: 2014 American Control Conference, pp. 4702-4707.

Ramezani H, Jamali-Rad H and Leus G (2013) Target localization and tracking for an isogradient sound speed profile. IEEE Transactions on Signal Processing 61(6): 1434-1446.

Rao CR (2008) Linear Statistical Inference and its Applications. 2nd ed. New York: John Wiley \& Sons, Inc.
Tan YT, Gao R and Chitre M (2014) Cooperative path planning for range-only localization using a single moving beacon. IEEE Journal of Oceanic Engineering 39(2): 371-385.

Trees HLV, Bell KL and Tian Z (2013) Detection Estimation and Modulation Theory, Part I: Detection, Estimation, and Filtering Theory. 2nd ed. New York: John Wiley \& Sons, Inc.

Ucinski D (2004) Optimal Measurement Methods for Distributed Parameter System Identification. Boca Raton, FL: CRC Press.

Vaganay J, Baccou P and Jouvencel B (2000) Homing by acoustic ranging to a single beacon. In: OCEANS 2000 MTS/IEEE Conference and Exhibition. Conference Proceedings (Cat. No.00CH37158), Vol. 2, pp. 1457-1462.

Vallicrosa G, Ridao P, Ribas D and Palomer A (2014) Active range-only beacon localization for AUV homing. In: 2014 IEEE/RSJ International Conference on Intelligent Robots and Systems, pp. 2286-2291.

Webster SE, Eustice RM, Singh H and Whitcomb LL (2012) Advances in single-beacon one-way-travel-time acoustic navigation for underwater vehicles. The International Journal of Robotics Research 31(8): 935-950.

Williams SB, Pizarro O, Steinberg DM, Friedman A and Bryson M (2016) Reflections on a decade of autonomous underwater vehicles operations for marine survey at the Australian Centre for Field Robotics. Annual Reviews in Control 42: 158-165. 